\title{
On the Order Dimension of Outerplanar Maps
}

\author{
Stefan Felsner \\ Institut für Mathematik \\ Technische Universität Berlin \\ felsner@math.tu-berlin.de
}

\author{
Johan Nilsson*† \\ MADALGO, BRICS \\ Department of Computer Science \\ University of Aarhus \\ johann@daimi.au.dk
}

\begin{abstract}
Schnyder characterized planar graphs in terms of order dimension. Brightwell and Trotter proved that the dimension of the vertex-edgeface poset $\mathbf{P}_{M}$ of a planar map $M$ is at most four. In this paper we investigate cases where $\operatorname{dim}\left(\mathbf{P}_{M}\right) \leq 3$ and also where $\operatorname{dim}\left(\mathbf{Q}_{M}\right) \leq 3$; here $\mathbf{Q}_{M}$ denotes the vertex-face poset of $M$. We show:

- If $M$ contains a $K_{4}$-subdivision, then $\operatorname{dim}\left(\mathbf{P}_{M}\right)=\operatorname{dim}\left(\mathbf{Q}_{M}\right)=4$.

- If $M$ or the dual $M^{*}$ contains a $K_{2,3}$-subdivision, then $\operatorname{dim}\left(\mathbf{P}_{M}\right)=4$. Hence, a map $M$ with $\operatorname{dim}\left(\mathbf{P}_{M}\right) \leq 3$ must be outerplanar and have an outerplanar dual. We concentrate on the simplest class of such maps and prove that within this class $\operatorname{dim}\left(\mathbf{P}_{M}\right) \leq 3$ is equivalent to the existence of a certain oriented coloring of edges. This condition is easily checked and can be turned into a linear time algorithm returning a 3-realizer.

Additionally, we prove that if $M$ is 2-connected and $M$ and $M^{*}$ are outerplanar, then $\operatorname{dim}\left(\mathbf{Q}_{M}\right) \leq 3$. There are, however, outerplanar maps with $\operatorname{dim}\left(\mathbf{Q}_{M}\right)=4$. We construct the first such example.
\end{abstract}

\section{Introduction}

This paper is about planar maps and the order dimension of posets related to them. A planar map $M=(G, D)$ consists of a finite planar multigraph $G$ and a plane drawing $D$ of $G$. By a planar map $M$ we mean the combinatorial data given by the set $V$ of vertices, the set $E$ of edges, the set $F$ of faces and the incidence relations between these sets.

The dual map $M^{*}$ of $M$ is defined as usual: there is a vertex $F^{*}$ in $M^{*}$ for each face $F$ in $M$, and an edge $e^{*}$ in $M^{*}$ for each edge $e$ of $M$, joining the dual vertices corresponding to the faces in $M$ separated by $e$ (if $e$ is a bridge, $e^{*}$ is a loop). Each vertex in $M$ will then correspond to a face of $M^{*}$.

Most of the maps we consider in this paper are outerplanar. We differentiate between two notions of outerplanar maps. A planar map $M=(G, D)$ is weakly outerplanar if $G$ is outerplanar, and strongly outerplanar if $G$ is

\footnotetext{
*Supported by the EU Research Training Network COMSTRU.

${ }^{\dagger}$ This research was done while the author visited TU Berlin.
} 
outerplanar and $D$ is an outerplane drawing of $G$, i.e., a plane drawing of $G$ where all the vertices are on the boundary of the outer face. When it is clear from the context, the qualifiers weakly and strongly will be omitted.

The dimension is a widely studied parameter of posets. Since its introduction by Dushnik and Miller [3] in 1941, dimension has moved into the core of combinatorics. There are close connections and analogies with the chromatic number of graphs and hypergraphs. From the applications point of view, dimension is attractive because low dimension warrants a small encoding complexity of the poset. Trotter [10] provides an extensive introduction to the area.

The vertex-edge-face poset $\mathbf{P}_{M}$ of a planar map $M$ is the poset on the vertices, edges and faces of $M$ ordered by inclusion. The vertex-face poset $\mathbf{Q}_{M}$ of $M$ is the subposet of $\mathbf{P}_{M}$ induced by the vertices and faces of $M$.

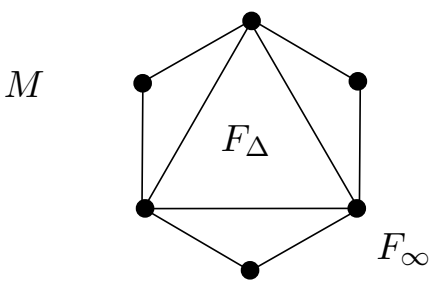

Figure 1: A planar map. Two faces are labeled, $F_{\infty}$ is the outer face.
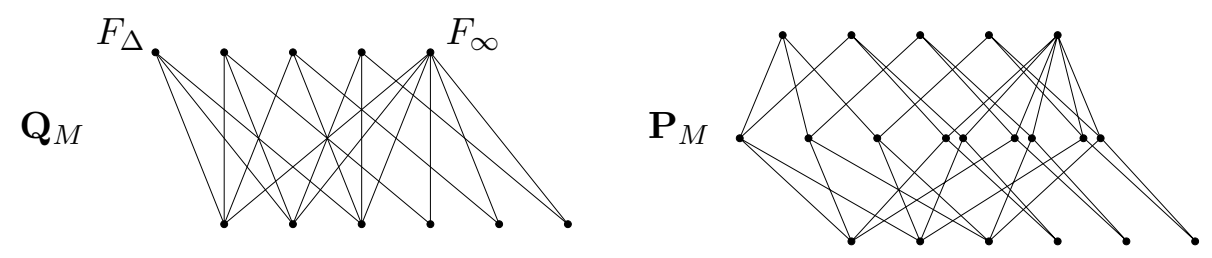

Figure 2: The vertex-face and the vertex-edge-face posets of the map from Figure 1

Note that if $M$ is connected, the vertex-edge-face poset $\mathbf{P}_{M^{*}}$ of the dual map is just the dual poset $\left(\mathbf{P}_{M}\right)^{*}$ (i.e., $x<y$ in $\left(\mathbf{P}_{M}\right)^{*}$ if and only if $y<x$ in $\left.\mathbf{P}_{M}\right)$. The same observation is true for $\mathbf{Q}_{M}$.

The dimension $\operatorname{dim}(\mathbf{P})$ of a poset $\mathbf{P}$ is the minimum number $t$ such that $t$ is the intersection of $t$ linear orders on the same ground set. From this it follows that if $Q$ is an induced subposet of $P$, then $\operatorname{dim}(\mathbf{Q}) \leq \operatorname{dim}(\mathbf{P})$. In particular this implies $\operatorname{dim}\left(\mathbf{Q}_{M}\right) \leq \operatorname{dim}\left(\mathbf{P}_{M}\right)$ for every map $M$.

The background to our investigations lies in Schnyder's characterization of planar graphs in terms of dimension [9] and in the two theorems of Brightwell and Trotter stated below. A simpler proof of Theorem 2 can be found in [5]. 
Theorem 1 (Brightwell and Trotter [2]). If $M$ is a planar map, then $\operatorname{dim}\left(\mathbf{P}_{M}\right) \leq 4$.

Theorem 2 (Brightwell and Trotter [1]). If $M$ is a 3-connected planar map, then $\operatorname{dim}\left(\mathbf{Q}_{M}\right)=4$.

It was observed by Li $\left[8\right.$ that the characterization of maps with $\operatorname{dim}\left(\mathbf{P}_{M}\right)=$ 2 given in the concluding section of [2] is incomplete but the full characterization exists, the paper is in preparation. The case of dimension is 2 is also well-understood algorithmically: There are fast algorithms to test whether a poset $\mathbf{P}$ is of dimension 2, see e.g. [7].

For $\operatorname{dim}\left(\mathbf{P}_{M}\right) \geq 3$ it is sufficient that $M$ has a vertex of degree 3. To test whether an arbitrary poset has dimension equal to 3 is known to be NP-complete [11]. A major open problem in the area is to determine the complexity of the dimension 3 problem for orders of height 2. Even the special case where the order of height 2 is the vertex-face poset of a planar map remains open. Motivated by these algorithmic questions we approach the problems of characterizing the maps $M$ with $\operatorname{dim}\left(\mathbf{P}_{M}\right) \leq 3$ and the maps with $\operatorname{dim}\left(\mathbf{Q}_{M}\right) \leq 3$. These characterization problems have earlier been posed by Brightwell and Trotter [2].

\subsection{Our contributions}

In Section 2 we prove that for $\operatorname{dim}\left(\mathbf{Q}_{M}\right) \leq 3$ it is necessary that $M$ is $K_{4^{-}}$ subdivision free. For $\operatorname{dim}\left(\mathbf{P}_{M}\right) \leq 3$ an additional necessary condition is that both $M$ and $M^{*}$ are $K_{2,3}$-subdivision free. This means that if $\operatorname{dim}\left(\mathbf{P}_{M}\right) \leq 3$, then both $M$ and $M^{*}$ are outerplanar.

In Section 3, we study the simplest class of maps $M$ such that $M$ and $M^{*}$ are outerplanar. We call these maps path-like. For maximal path-like maps we prove that $\operatorname{dim}\left(\mathbf{P}_{M}\right) \leq 3$ is equivalent to the existence of a special oriented coloring of the interior edges and characterize the path-like maps which admit such a coloring. The characterization is turned into a linear time algorithm that generates a 3-realizer, i.e., three linear extensions whose intersection is $\mathbf{P}_{M}$, or returns the information that $\operatorname{dim}\left(\mathbf{P}_{M}\right) \geq 4$.

Finally, in Section 4, we prove that if $M$ is 2-connected and $M$ and $M^{*}$ are outerplanar, then $\operatorname{dim}\left(\mathbf{Q}_{M}\right) \leq 3$. We also present a strongly outerplanar map with a vertex-face poset of dimension 4 . The example, a maximal outerplanar graph with 21 vertices, is quite large. We provide some arguments which indicate that our example is not far from being as small as possible.

\subsection{Tools from dimension theory}

In this section we recall some facts from the dimension theory of finite posets. The reader is referred to Trotter's monograph [10] for additional background and references. 
If $\mathbf{P}$ is a finite poset, a family $\mathcal{R}=\left\{L_{1}, L_{2}, \ldots, L_{t}\right\}$ of linear extensions of $\mathbf{P}$ is called a realizer of $\mathbf{P}$ if $P=\cap \mathcal{R}$, i.e. $x<y$ in $\mathbf{P}$ if and only if $x<y$ in $L$ for all $L \in \mathcal{R}$. The dimension of $\mathbf{P}$ is the minimum cardinality of a realizer of $\mathbf{P}$.

A critical pair is a pair of incomparable elements $(a, b)$ such that $x<b$ if $x<a$ and $y>a$ if $y>b$ for all $x, y \in \mathbf{P}$. A family of linear extensions $\mathcal{R}$ of $P$ is a realizer of $\mathbf{P}$ if and only if each critical pair $(a, b)$ is reversed in some linear extension $L \in \mathcal{R}$, i.e., $b<a$ in $L$. An incomparable min-max pair, i.e., a pair of incomparable elements $(a, b)$ where $a$ is a minimal element and $b$ is a maximal element of $\mathbf{P}$, is always critical.

An alternating cycle is a sequence of critical pairs $\left(a_{0}, b_{0}\right), \ldots,\left(a_{k}, b_{k}\right)$ such that $\left.a_{i}<b_{(i+1} \bmod (k+1)\right)$ for all $i=0, \ldots, k$. A fundamental result is that $\operatorname{dim}(\mathbf{P}) \leq t$ if and only if there exists a $t$-coloring of the critical pairs of $\mathbf{P}$ such that no alternating cycle is monochromatic.

In the following example we illustrate how these facts can be combined to determine the dimension of a specific incidence order.

Example: Let $M$ be the planar map of the complete graph $K_{4}$. Every vertex has a single non-incident face, hence, there are these four incomparable min-max pairs in $\mathbf{Q}_{M}$. These are all the critical pairs. Any two of these critical pairs form an alternating cycle. Therefore, the hypergraph of alternating cycles is again a $K_{4}$ and has chromatic number 4 . This shows that $\operatorname{dim}\left(\mathbf{Q}_{M}\right)=4$.

\section{Vertex-edge-face posets of dimension at most 3}

From Theorem 2, we know that $\operatorname{dim}\left(\mathbf{Q}_{M}\right)=4$ for every 3-connected map $M$. We show that this excludes $K_{4}$-subdivisions from being contained in $M$ if $\operatorname{dim}\left(\mathbf{Q}_{M}\right) \leq 3$.

Theorem 3. Let $M$ be a planar map that contains a subdivision of $K_{4}$. Then $\operatorname{dim}\left(\mathbf{Q}_{M}\right)>3$.

Proof. We will prove that $\mathbf{Q}_{M}$ has the vertex-face poset of some 3-connected planar map as a subposet, and then apply the Brightwell-Trotter Theorem. This is essentially done in two steps: first 1-vertex cuts and then 2-vertex cuts are removed.

A $K_{4}$-subdivision in $M$ will be contained in a 2-connected component of $M$. The vertex-face poset of a 2 -connected component of $M$ is an induced subposet of $\mathbf{Q}_{M}$. Hence, we can assume that $M$ is 2-connected.

Now, consider a 2-vertex cut $\{x, y\}$. There must be two components $C_{1}$ and $C_{2}$ such that removing $x$ and $y$ separates $C_{1}$ from $C_{2}$. We create two new maps by replacing one of the two components by an edge $\{x, y\}$, see Figure 3. The vertex-face posets of these new maps are subposets of $\mathbf{Q}_{M}$. 


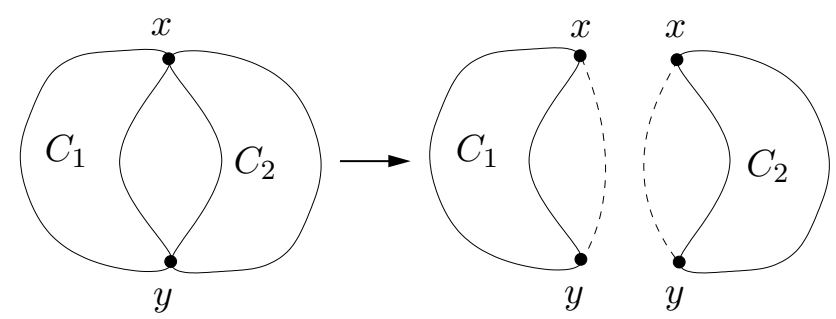

Figure 3: Removing a separating pair.

Furthermore, if $M$ contains a $K_{4}$-subdivision, one of the new maps must contain a $K_{4}$-subdivision.

For vertex-edge-face posets, we provide another criterion which forces dimension 4. If $M$ contains a $K_{2,3}$-subdivision, then $\operatorname{dim}\left(\mathbf{P}_{M}\right)=4$. Before analyzing the general situation we deal with the simple case where $M$ is, actually, a subdivision of $K_{2,3}$.

Proposition 4. Let $M$ be a planar drawing of a subdivision of $K_{2,3}$. Then $\operatorname{dim}\left(\mathbf{P}_{M}\right)>3$.

Proof. Let $x$ and $y$ be the two vertices of degree 3 in $M$, and let $P_{1}, P_{2}$ and $P_{3}$ be the three $x-y$ paths. The map has three faces $F_{1}, F_{2}$ and $F_{3}$. The vertex closest to $y$ in the path $P_{i}$ is denoted $v_{i}$, see Figure 4.

Suppose $\left\{L_{1}, L_{2}, L_{3}\right\}$ is a realizer of $\mathbf{P}_{M}$. By symmetry, we may assume that $y>x$ in $L_{1}$ and $L_{2}$ and $x>y$ in $L_{3}$. The edge $\left\{v_{1}, y\right\}$ can go below $x$ only in $L_{3}$. In $L_{3}$ we thus have $v_{1}$ below $\left\{v_{1}, y\right\}$ below $x$ below $F_{1}, F_{2}$ and $F_{3}$. In the same way, we obtain that $v_{2}$ and $v_{3}$ are below $F_{1}, F_{2}$ and $F_{3}$ in $L_{3}$. Hence, none of the three critical pairs of the the subposet $\mathbf{S}$ induced by $v_{1}, v_{2}, v_{3}, F_{1}, F_{2}$ and $F_{3}$ in $\mathbf{P}_{M}$ can be reversed in $L_{3}$. However, $\mathbf{S}$ is a crown with $\operatorname{dim}(\mathbf{S})=3$. This shows that $\left\{L_{1}, L_{2}, L_{3}\right\}$ is not a realizer of $\mathbf{P}_{M}$. Hence, $\operatorname{dim}\left(\mathbf{P}_{M}\right)>3$.

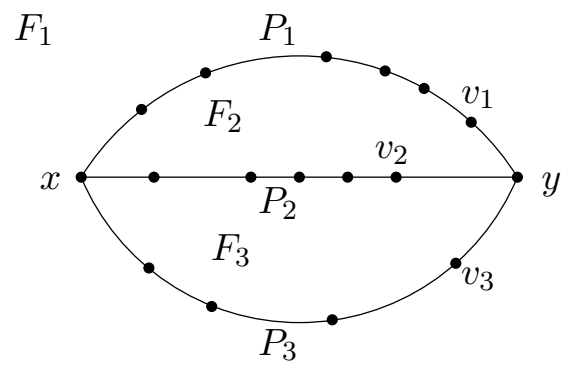

Figure 4: The three paths $P_{1}, P_{2}$ and $P_{3}$ partition the map into 3 regions. 
For the general case, where $M$ only contains a $K_{2,3}$-subdivision, we have to use a more sophisticated technique. We illustrate this technique with an alternative proof of the simple case.

Second proof of Proposition 4. The vertices and edges of path $P_{i}$ all belong to $F_{i} \cap F_{i+1}$ (cyclically). Hence, each path $P_{i}$ induces a fence of the form $x<e_{0}>u_{1}<e_{1}>\ldots>u_{s}<e_{s}>y$ between $x$ and $y$ in $\mathbf{P}_{M}$ such that all maximal elements are below $F_{i}$ and $F_{i+1}$. These three fences are mutually disjoint except for $x$ and $y$.

Suppose $\left\{L_{1}, L_{2}, L_{3}\right\}$ is a realizer of $\mathbf{P}_{M}$. By symmetry, we may assume that $y>x$ in $L_{1}$ and $L_{2}$ and $x>y$ in $L_{3}$. Now, consider the fence induced by $P_{i}, i \in 1,2,3$; see Figure 5 .

The edge $\left\{v_{i}, y\right\}$ must be below $x$ in $L_{3}$, hence $v_{i}$ is below $x$ in $L_{3}$. Let $w_{i}$ be the last vertex encountered when traversing the path $P_{i}$ from $y$ to $x$ which is below $x$ in $L_{3}$, and let $e_{i}$ be the edge leaving $w_{i}$ in direction of $x$. The choice of $w_{i}$ implies that $e_{i}$ is above $x$ and $y$ in $L_{3}$. Since $e_{i}$ has to go below $y$ somewhere there is an index $j_{i} \in\{1,2\}$ such that $e_{i}$ and thus $w_{i}$ go below $y$ in $L_{j_{i}}$.

Two of the three indices $j_{1}, j_{2}, j_{3}$ must be equal, so we can w.l.o.g. assume that $w_{1}$ and $w_{2}$ are below $y$ in $L_{2}$. Recalling that $w_{1}$ and $w_{2}$ are below $x$ in $L_{3}$ we conclude that $w_{1}$ and $w_{2}$ are below all faces $F_{j}$ in $L_{2}$ and in $L_{3}$.

Now, none of the critical pairs of the subposet $\mathbf{2}+\mathbf{2}$ of $\mathbf{P}_{M}$ induced by $w_{1}$, $w_{2}, F_{1}$ and $F_{3}$ are reversed in $L_{2}$ or $L_{3}$. But $\operatorname{dim}(\mathbf{2 + 2})=2$, so the critical pairs of $\mathbf{Q}$ cannot be reversed in $L_{1}$ alone. Hence $\left\{L_{1}, L_{2}, L_{3}\right\}$ cannot be a realizer of $\mathbf{P}_{M}$.

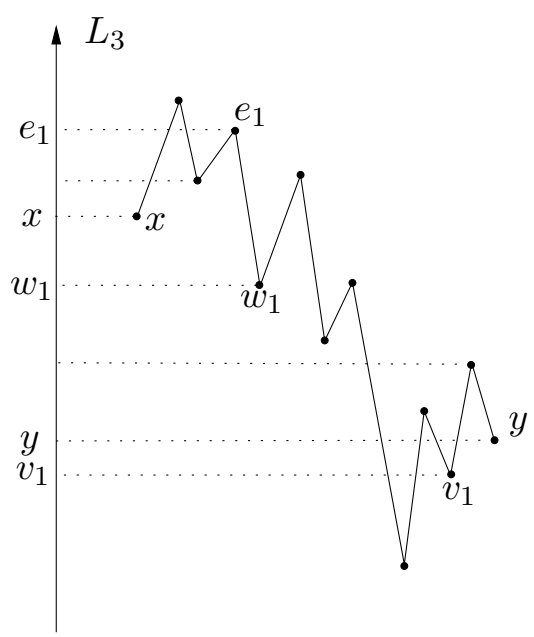

Figure 5: The fence of path $P_{1}$ and $L_{3}$.

We now move on to the slightly more complicated case where $M$ only contains a subdivision of $K_{2,3}$. 
Theorem 5. Let $M$ be a planar map such that $M$ contains a subdivision of $K_{2,3}$. Then $\operatorname{dim}\left(\mathbf{P}_{M}\right)>3$.

Proof. If $M$ contains a subdivision of $K_{4}$, the conclusion of the lemma follows from Theorem 3. We thus can assume that $M$ contains no subdivision of $K_{4}$.

Let $x$ and $y$ be the degree 3 vertices in the subdivision of $K_{2,3}$. Our goal is to find at least three mutually disjoint fences $\mathbf{T}_{i}$ between $x$ and $y$, and a set of faces $F_{i}$ such that $x, y \in F_{i}$ and each minimal element in $\mathbf{T}_{i}$ is below $F_{i}$ and $F_{i+1}$.

Given fences $\mathbf{T}_{i}$ and faces $F_{i}$ as described we can continue as in the previous proof: Assume a realizer $\left\{L_{1}, L_{2}, L_{3}\right\}$ such that $y>x$ in $L_{1}$ and $L_{2}$ and $x>y$ in $L_{3}$. In each fence $\mathbf{T}_{i}$ we find a minimal element $w_{i}$ which is below $x$ in $L_{3}$ and below $y$ in some $L_{j_{i}}, j_{i} \in\{1,2\}$. Since $i \geq 3$ there are indices $a$ and $b$ with $j_{a}=j_{b}$, and we can w.l.o.g. let $j_{a}=j_{b}=1$. Let $a^{\prime} \in\{a, a+1\}$ and $b^{\prime} \in\{b, b+1\}$ be such that $w_{b} \notin F_{a^{\prime}}$ and $w_{a} \notin F_{b^{\prime}}$. Hence, $w_{a}, F_{a^{\prime}}, w_{b}, F_{b^{\prime}}$ induce a $\mathbf{2 + 2}$. The critical pairs of this $\mathbf{2 + 2}$ are not reversed in $L_{3}$ nor in $L_{1}$, and they can't both be reversed in $L_{2}$. This is in contradiction to the assumption that $\left\{L_{1}, L_{2}, L_{3}\right\}$ is a realizer. Hence, $\operatorname{dim}\left(\mathbf{P}_{M}\right)>3$.

It remains to show how to determine appropriate fences $\mathbf{T}_{i}$. Consider a maximal set $P_{0}, P_{1}, \ldots, P_{k}$ of pairwise internally disjoint paths from $x$ to $y$. Clearly, $k \geq 2$. Choose the numbering corresponding to the cyclic order of their first edges at $x$. For $i=1, . ., k+1$ let $R_{i}$ be the bounded area between $P_{i-1}$ and $P_{i}$, with $P_{k+1}=P_{0}$.

Claim A. If $M$ contains no subdivision of $K_{4}$, then every region $R_{i}$ contains a face $F_{i}$ with $x$ and $y$ and interior vertices of $P_{i-1}$ and $P_{i}$ on its boundary.

The maximality of the family $P_{0}, P_{1}, \ldots, P_{k}$ implies that in $R_{i}$ there is a face $F_{i}$ that has a nonempty intersection with the interior of both $P_{i-1}$ and $P_{i}$. Next we prove that this face $F_{i}$ contains $x$ and $y$. Otherwise, the cycle consisting of $P_{i-1}$ and $P_{i}$ has a chordal path separating $x$ from $y$. This path, together with $P_{i-1}, P_{i}$ and some $P_{j}, j \notin\{i-1, i\}$ is a subdivision of $K_{4}$ in $M$.

Since a subdivision of $K_{4}$ already implies that $\operatorname{dim}\left(\mathbf{P}_{M}\right)=4$ we continue with the assumption that there is a face $F_{i}$ with the specifiesd properties for all $i$.

Let $u$ and $w$ be vertices of $P_{i}$ such that $u$ is closer to $x$ than $w$ and $(u, w) \neq(x, y)$. A shortcut between $u$ and $w$ over $P_{i}$ is a path from $u$ to $w$ which is internally disjoint from $P_{i}$. Two shortcuts, $\left\{u_{1}, w_{1}\right\}$ and $\left\{u_{2}, w_{2}\right\}$ are crossing if their order along $P_{1}$ is either $u_{1}, u_{2}, w_{1}, w_{2}$ or $u_{2}, u_{1}, w_{2}, w_{1}$. In particular this requires the four vertices to be pairwise different.

Claim B. There is no crossing pair of shortcuts on $P_{i}$. 


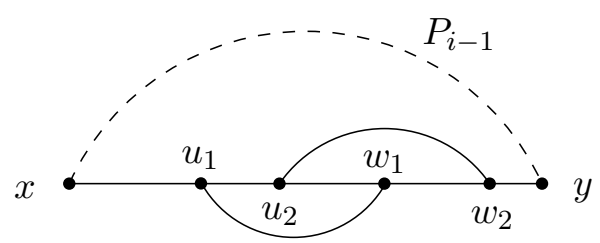

Figure 6: Crossing shortcuts.

Otherwise, the four vertices of the two shortcuts are the degree three vertices of a subdivision of $K_{4}$. This subdivision of $K_{4}$ is formed by the shortcuts together with $P_{i}$ and $P_{i-1}$. See Figure 6

Let $V_{i}$ be the set of all vertices of $P_{i}$ that are contained in $F_{i} \cap F_{i+1}$.

Claim C. Two consecutive vertices $u$ and $w$ in $V_{i}$ either are the two endpoints of an edge or there exists a face $F$ such that $F \cap V_{i}=\{u, w\}$.

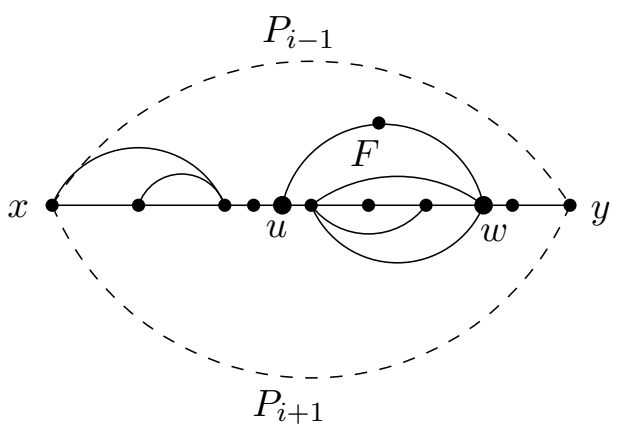

Figure 7: The common face $F$ of $u$ and $w$.

Suppose $\{u, w\}$ is not an edge. From Claim B it follows that there is a shortcut $\{u, w\}$ over $P_{i}$. Essentially the same proof as for Claim A shows that the subregion bounded by $P_{i}$ and the shortcut between $u$ and $w$ contains a face $F$ with $u, w \in F$; otherwise, there is a $K_{4}$-subdivision.

The fence $\mathbf{T}_{i}$ consists of $V_{i}$ (the set of minimal elements) and edges, respectively faces, over consecutive pairs of vertices in $V_{i}$. The existence of a $K_{2,3}$-subdivision between $x$ and $y$ implies that at least three of the fences $\mathbf{T}_{0}, \mathbf{T}_{1}, \ldots, \mathbf{T}_{k}$ are nontrivial, i.e., have minima different from $x$ and $y$. These fences can be used to conclude the proof.

Theorem 6. If $\operatorname{dim}\left(\mathbf{P}_{M}\right) \leq 3$, then $M$ and $M^{*}$ are both weakly outerplanar.

Proof. From Theorem 3 and Theorem [5, we know that if $\operatorname{dim}\left(\mathbf{P}_{M}\right) \leq 3$ then $M$ contains neither a $K_{4}$-subdivision nor a $K_{2,3}$-subdivision. This is equivalent to saying that the graph $G$ corresponding to the map $M$ is outerplanar. Since $\mathbf{P}_{M}$ and $\mathbf{P}_{M^{*}}$ are dual orders and, hence have the same dimension, the same necessary condition for $\operatorname{dim}\left(\mathbf{P}_{M}\right) \leq 3$ applies to $M^{*}$. 
Note that testing if $M$ and $M^{*}$ are weakly outerplanar, i.e., if the corresponding graphs are outerplanar, can be done in linear time [6].

\section{Path-like maps and permissible colorings}

From Theorem 6 we know that if $\operatorname{dim}\left(\mathbf{P}_{M}\right) \leq 3$, both $M$ and $M^{*}$ are weakly outerplanar. In this section we study the order dimension of 2-connected maps $M$, such that $M$ is strongly outerplanar and $M^{*}$ is weakly outerplanar.

A 2-connected component of an outerplanar map $M$ has a Hamilton cycle. If the graph of $M$ is simple, the Hamilton cycle is unique. This yields a natural partition of the edges of $M$ into cycle edges and chordal edges. The restriction of the dual graph to the graph induced by the vertices corresponding to bounded faces is called the interior dual. For a strongly outerplanar map, the edges of the interior dual are just the dual edges of the chordal edges.

We say that a simple 2-connected outerplanar map $M$ is path-like if and only if the interior dual of $M$ is a simple path. Note that this implies that the Hamilton cycle is the boundary of the outer face $F_{\infty}$, i.e. that $M$ is strongly outerplanar. Since the interior dual is a path, it follows that $M^{*}$ is weakly outerplanar. On the other hand, if $M$ is a 2-connected strongly outerplanar map and $M^{*}$ is weakly outerplanar, the interior dual of $M$ must be a simple path. Hence, $M$ is path-like if and only if $M$ is a 2-connected outerplanar map such that $M^{*}$ is weakly outerplanar.

From Theorem [6 it follows that if $M$ is a 2-connected strongly outerplanar map with $\operatorname{dim}\left(\mathbf{P}_{M}\right) \leq 3, M$ must be path-like. We can also prove something slightly stronger.

Proposition 7. Let $M$ be a simple 2-connected planar map with $\operatorname{dim}\left(\mathbf{P}_{M}\right) \leq$ 3. The map $M^{\prime}$ obtained by moving all the chordal edges of $M$ into the interior of the Hamilton cycle is path-like.

Proof. Suppose not. Then the interior dual of $M^{\prime}$ contains a vertex of degree at least 3 , and hence its dual $\left(M^{\prime}\right)^{*}$ contains a subdivision of $K_{2,3}$ with the dual of one degree 3 vertex inside the Hamilton cycle $H$ and the dual of the other outside. We proceed to show that we can move the necessary chordal edges outside, one by one, to create $M$ without destroying the $K_{2,3^{-}}$ subdivision in the dual.

We do this as follows: let $M^{\prime}=M_{0}, M_{1}, \ldots, M_{k}=M$ be a sequence of maps such that $M_{j+1}$ is obtained from $M_{j}$ by moving a chordal edge from the inside to the outside of the Hamilton cycle. The proposition is implied by the following lemma.

Lemma 8. For each map $M_{i}, i=0,1, \ldots, k$, the dual map $M_{i}^{*}$ contains a $K_{2,3}$-subdivision such that $H^{*}$ separates the two vertices of degree 3. 
Proof. We prove the lemma by induction on $i$. We have already seen that the statement is true for $M_{0}$.

Suppose the statement is true for $M_{i}$. Let $e=\{u, v\}$ be the edge that has to be moved to the outside of $H$ to get from $M_{i}$ to $M_{i+1}$. Let $F^{*}$ and $G^{*}$ be the degree 3 vertices in a $K_{2,3}$-subdivision in $M_{i}^{*}$, where $F$ is inside $H$. We construct a new map $M_{i}^{\prime}$, by adding the edge $e^{\prime}=\{u, v\}$ to $M_{i}$ outside $H$, see Figure 8 . Note that $F$ and $G$ must be on the same side of the cycle $\left\{e, e^{\prime}\right\}$, since otherwise $\left\{e^{*},\left(e^{\prime}\right)^{*}\right\}$ is a 2-edge cut in $\left(M_{i}^{\prime}\right)^{*}$ separating $F^{*}$ from $G^{*}$; this is impossible as $F^{*}$ and $G^{*}$ are the degree 3 vertices in a $K_{2,3}$-subdivision.

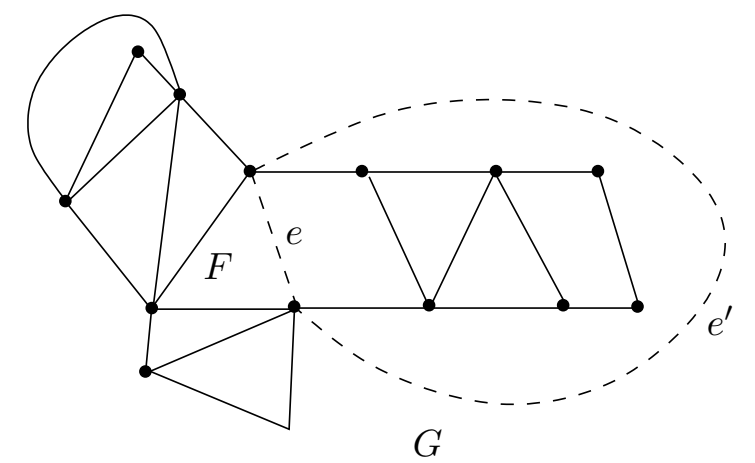

Figure 8: The map $M_{i}^{\prime}$ is constructed by adding $e^{\prime}$ to $M_{i}$.

Claim A. Let $P^{*}$ be a simple $F^{*}-G^{*}$ path in $\left(M_{i}^{\prime}\right)^{*}$. If $e^{*} \in P^{*}$, then $P^{*}$ consists of at least 3 edges.

Suppose $e^{*} \in P^{*}$. Since $P^{*}$ is simple, and $F$ and $G$ are on the same side of the cycle $\left\{e, e^{\prime}\right\}$, it follows that $\left(e^{\prime}\right)^{*} \in P^{*}$. Moreover, the dual $H^{*}$ of the Hamilton cycle $H$ separates $F^{*}$ from $G^{*}$, so $P^{*}$ must contain the dual of a cycle edge. But neither $e$ nor $e^{\prime}$ are cycle edges, so the claim follows.

Now, $M_{i+1}$ is obtained by removing $e$ from $M_{i}^{\prime}$. In $\left(M_{i}^{\prime}\right)^{*}$, this corresponds to the contraction of edge $e^{*}$. If $e^{*}$ is not on any $F^{*}-G^{*}$ path, then $M_{i+1}^{*}$ contains a $K_{2,3}$-subdivision. On the other hand, an $F^{*}-G^{*}$ path containing $e^{*}$ has at least 3 edges, hence, the contraction of $e^{*}$ cannot destroy the $K_{2,3^{-}}$ subdivision. This completes the proof of the lemma and the proposition.

For later reference we restate the essence of the proof:

Corollary 9. Let $M^{\prime}$ be obtained from a simple weakly outerplanar map $M$ by flipping all chordal edges to the interior of the Hamilton cycle. If $\left(M^{\prime}\right)^{*}$ contains a $K_{2,3}$-subdivision, then so does $M^{*}$.

In the rest of this section, we consider maximal path-like maps, i.e., pathlike maps where all interior faces are triangles. Consider a triangle of a 
maximal path-like map $M$. Each of the three vertices forms a critical pair with a face or edge that is above the other two vertices of the triangle. In Figure 9 these critical pairs are $\left(u, F_{u}\right),\left(v, F_{v}\right),\left(w, e_{w}\right)$. Note that the critical pairs associated to a triangle are in bijection to its angles.

Suppose $\operatorname{dim}\left(\mathbf{P}_{M}\right)=3$ and $L_{1}, L_{2}, L_{3}$ is a realizer. From this we obtain a 3-coloring of the of inner angles of the map: For every angle choose a color $i$ such that the critical pair corresponding to the angle is reversed in $L_{i}$. For convenience we interchangably use 1, 2, 3 and red, green, blue as names for the three colors in the angle coloring.

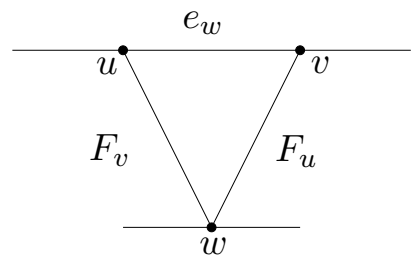

Figure 9: The critical pairs of a triangle.

We go on to prove some properties of such an angle 3-coloring for a maximal path-like map $M$ with $\operatorname{dim}\left(\mathbf{P}_{M}\right) \leq 3$. In the following we will use $F_{u}$ as the default name for the edge or face in the critical pair with $u$. For example in the proof of the next lemma at least one of $F_{u}, F_{v}, F_{w}$ must be an edge since the map $M$ is path like.

Lemma 10. No two angles in a triangle can have the same color.

Proof. Consider a triangle with the angle coloring described above. Any two of the critical pairs $\left(u, F_{u}\right),\left(v, F_{v}\right),\left(w, F_{w}\right)$ form an alternating cycle. Hence, no two pairs can be reversed in the same linear extension.

Lemma 11. Let $e=\{a, b\}$ be a chordal edge. The four angles $\alpha_{\ell}, \alpha_{r}, \beta_{\ell}$ and $\beta_{r}$ incident on $e$ at $a$ and $b$ are colored such that all three colors are used, and one of the pairs $\left(\alpha_{\ell}, \alpha_{r}\right)$ or $\left(\beta_{\ell}, \beta_{r}\right)$ is monochromatic.

Proof. We refer to Figure 10, Suppose $\alpha_{\ell}=1$ and $\alpha_{r}=2$. This implies that in $L_{1}$ we have $F_{b}^{\ell}$ and $F_{b}^{r}$ above $a$ above $F_{a}^{\ell}$ above $b$. In $L_{2}$ we have the same order with $F_{a}^{r}$ taking the role of $F_{a}^{\ell}$. Hence $b$ has to be above both $F_{b}^{\ell}$ and $F_{b}^{r}$ in $L_{3}$ which is equivalent to $\beta_{\ell}=\beta_{r}=3$.

Suppose both pairs of angles have the same colors, say $\alpha_{\ell}=\alpha_{r}=1$ and $\beta_{\ell}=\beta_{r}=2$ Then the third angle in both triangles (at $x$ and $y$, respectively) must have color 3 . This induces a monochromatic alternating cycle $\left(x, F_{x}\right)$, $\left(y, F_{y}\right)$, a contradiction.

By Lemma 11 we can encode the angle coloring as an oriented coloring of the chordal edges: each chordal edge gets the color that appears twice around it and is oriented towards the endpoint where this happens. 


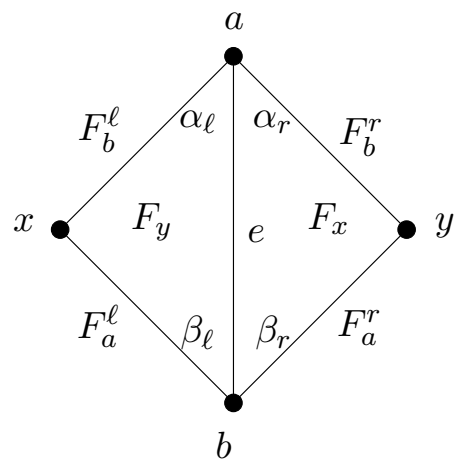

Figure 10: Colors and critical pairs around a chordal edge.

The orientation of an interior triangle is either clockwise or counterclockwise depending on the cyclic reading which shows the colors 1,2,3 in this order. Lemma 10 implies that the orientation of interior triangles is defined.

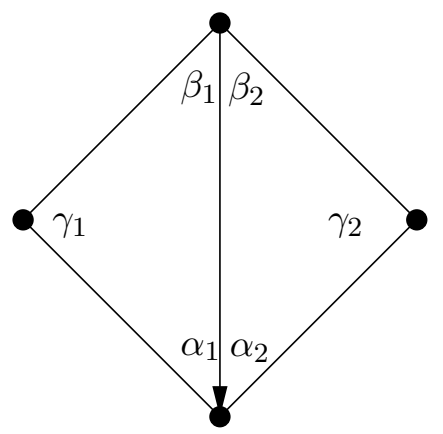

Figure 11: The triangles must have the same orientation.

Lemma 12. All interior triangles have the same orientation.

Proof. This is a direct consequence of Lemma 11 and Lemma 10, Referring to Figure 11 we discuss one of the cases: Suppose the left triangle in the figure is counterclockwise, i,e., $\left(\alpha_{1}, \beta_{1}, \gamma_{1}\right)=(i, i+1, i+2)$. The orientation of the edge implies $\alpha_{2}=i$, with Lemma 11 we get $\beta_{2}=i+2$. This shows that the right triangle is counterclockwise as well.

Lemma 13. If $c$ is the color of a chordal edge $e$, then $e>F_{\infty}$ in $L_{c}$.

Proof. Again referring to Figure 11 we observe that $\gamma_{1} \neq \gamma_{2}$ and $c \neq \gamma_{1}, \gamma_{2}$ by Lemma 12. Hence, $e<F_{\infty}$ in $L_{\gamma_{1}}$ and $L_{\gamma_{2}}$. Therefore $e>F_{\infty}$ in $L_{c}$.

Lemma 14. Each vertex is either a sink or a source w.r.t. the orientation of the chordal edges. 


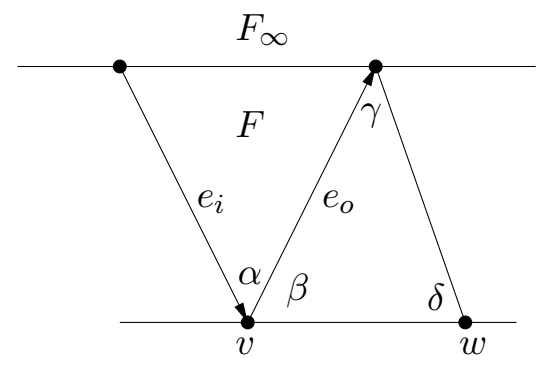

Figure 12: A vertex must be either a sink or a source.

Proof. Suppose that there is a triangle $F$ and a vertex $v$ such that $F$ has two chordal edges $e_{i}$ and $e_{o}$ meeting at $v$, such that $e_{i}$ is incoming and $e_{o}$ is outgoing at $v$. See Figure 12. The colors of the angles $\alpha, \beta$ and $\gamma$ must be pairwise different by Lemma 11. Hence, $\alpha$ and $\delta$ must have the same color (Lemma 10). But $\alpha$ has the same color as $e_{i}$. From Lemma 13$]$ it follows that the alternating cycle $\left(e_{i}, F_{\infty}\right),(w, F)$ is monochromatic - contradiction.

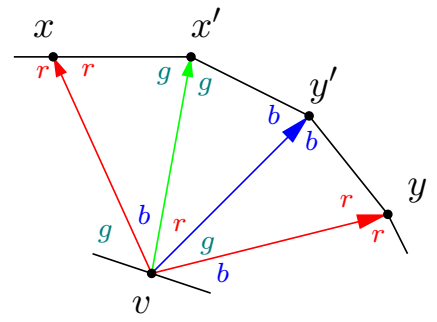

Figure 13: No two outgoing edges can have the same color.

Proposition 15. No two outgoing edges from a vertex have the same color.

Proof. Suppose that $v$ has two outgoing edges of the same color and that all triangles are clockwise. From the coloring of angles it follows that edges sharing an angle have different colors. Even more, the colors of the outgoing edges at $v$ in clockwise order cycle through $1,2,3$. Hence, we find a sequence $x, x^{\prime}, y^{\prime}, y$ of vertices, such that $v x$ and $v y$ have the same color, see Figure 13 . Now, $v$ is above $x$ in colors green and blue, so any face incomparable to $x$ which contains $v$ has to be below $x$ in red. The same is true for $y$. In particular $x>\left\{v, y^{\prime}, y\right\}$ and $y>\left\{v, x, x^{\prime}\right\}$ in red. This is a monochromatic red alternating cycle - contradiction.

Corollary 16. No vertex has four or more outgoing chordal edges.

We say that the colors of the chordal edges bounding a face are the colors of the face. A face with two colors is called bicolored. 
Proposition 17. No two bicolored faces have the same colors.

Proof. Suppose $F$ and $F^{\prime}$ are two such faces. Suppose the two colors are red and green. Then $F_{\infty}$ is below $F$ and $F^{\prime}$ in red and green (Lemma 13). Therefore, $F$ and $F^{\prime}$ can be below any vertex only in blue. Let $x$ be vertex in $F \backslash F^{\prime}$ and $y$ be a vertex in $F^{\prime} \backslash F$. Then $\left(x, F^{\prime}\right),(y, F)$ is a monochromatic blue alternating cycle - contradiction.

We say that an oriented coloring satisfying Lemma 12, Proposition 15 and Proposition 17 is permissible. The map of Figure 14 is shown with a permissible coloring. We call it the canonical map. The vertices (edges) in the top of the figure are called $q$-vertices (edges) and the ones in the bottom of the figure are called $p$-vertices. Note that faces $F_{2}, F_{4}$ and $F_{6}$ are bicolored.

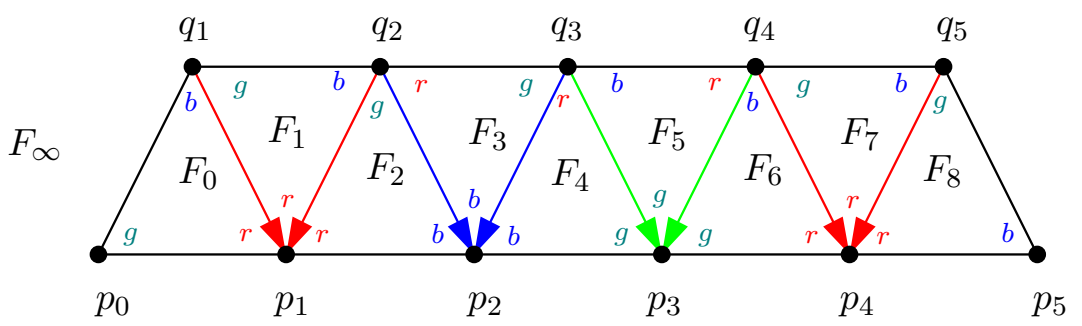

Figure 14: The canonical map.

Lemma 18. Any maximal path-like map with a permissible coloring of the chordal edges can be constructed from the canonical map by a sequence of the following three operations:

(i) Contracting one of the q-edges $\left\{q_{2}, q_{3}\right\}$ or $\left\{q_{3}, q_{4}\right\}$.

(ii) Subdividing a q-edge. Chordal edges between the new vertices and the p-vertex in the triangle are inserted with the same color and orientation as the old edges.

(iii) Deleting all the vertices, edges and faces on one side of a chordal edge.

Proof. Let $M$ be a maximal path-like map with a permissible coloring of the chordal edges. If $M$ has $n$ sinks, then there are $n-1$ bicolored faces in $M$. From Proposition 17 and Lemma 12 it follows that the canonical map has the maximum possible number of sinks.

Again from Proposition 17 it follows that $M$ has at most one vertex with outdegree 3 . We can contract $\left\{q_{2}, q_{3}\right\}$ or $\left\{q_{3}, q_{4}\right\}$ in the canonical map to get such a vertex. Note that contracting $\left\{q_{2}, q_{3}\right\}$ and $\left\{q_{3}, q_{4}\right\}$ would yield a map with a vertex of out-degree four, i.e., a map that has no permissible coloring. 
Any sinks or sources in the canonical map that are not in $M$ can be removed using operation (iii). Hence, what remains is possibly to add some sources of degree one to the map $M^{\prime}$ we have constructed. But this is easy, since the new inserted chordal edges must have the same colors as the faces they split, i.e., we can just subdivide the $q$-edges as in (ii).

Lemma 19. Let $M$ be the canonical map. Then $\operatorname{dim}\left(\mathbf{P}_{M}\right)=3$.

Proof. Consider the vertical symmetry through $q_{3}$ and the edge $\left\{p_{2}, p_{3}\right\}$. This partitions the vertex set into a left part $V_{l}=\left\{p_{0}, p_{1}, p_{2}, q_{1}, q_{2}\right\}$ a right part $V_{r}=\left\{p_{5}, p_{4}, p_{3}, q_{5}, q_{4}\right\}$ and $\left\{q_{3}\right\}$.

We construct three partial orders, one for each color. We start with the order of the vertices. In the green order we want $V_{r}>q_{3}>V_{l}$. The ordering of the vertices in $V_{l}$ and $V_{r}$ is such that it allows to reverse all critical pairs corresponding to green angles, i.e., on $V_{l}: p_{0}>q_{1}>q_{2}>\left\{p_{2}, p_{1}\right\}$ and on $V_{r}: p_{3}>q_{4}>q_{5}>\left\{p_{4}, p_{5}\right\}$. We add the relations $p_{2}>p_{1}$ and $p_{5}>p_{4}$ so that the order on $V_{l}$ and $V_{r}$ conforms with the clockwise ordering around the outer face with start in $p_{0}$ and $p_{3}$, respectively.

The blue partial order is created symmetrically. I.e., it is obtained from the green order with the mappings $p_{i} \rightarrow p_{5-i}$ and $q_{j} \rightarrow q_{6-j}$. Note that the order on $V_{l}$ and $V_{r}$ conforms with the counterclockwise ordering around the outer face with start in $p_{2}$ and $p_{5}$, respectively.

For the red partial order, we construct two linear orders on $V_{l} \cup\left\{q_{3}\right\}$ and $V_{r} \cup\left\{q_{3}\right\}$. These linear orders gives us a partial order on $V_{l} \cup V_{r} \cup$ $\left\{q_{3}\right\}$. In the linear order on $V_{l} \cup\left\{q_{3}\right\}$, the vertices come in the clockwise ordering around the outer face boundary with $p_{1}$ as maximal element, i.e, $p_{1}>p_{0}>q_{1}>q_{2}>q_{3}>p_{2}$. The right part is done symmetrically, $p_{4}>p_{5}>q_{5}>q_{4}>q_{3}>p_{3}$.

We now have three partial orders on the vertices. We extend these to partial orders on the vertices, edges and faces in three steps. First, we insert the Hamilton cycle edges and the outer face as low as possible in each of the three orders. Then the chordal edges are put above the outer face in their color, and as low as possible in the other two colors. Finally, the interior faces are inserted as low as possible.

Claim R. Every critical pair is reversed in one of the partial orders.

The lemma clearly follows from this claim; any three linear extensions of the partial orders constructed will then form a realizer.

There are three types of critical pairs: edge-face pairs, vertex-edge pairs and vertex-face pairs. All edge-face pairs are of the form (chordal edge, outer face), so they are reversed in the color of the edge.

Consider a vertex-edge critical pair $(v, e)$. For the pair to be critical $v$ and $e$ must belong to a triangle and $e$ has to be an edge of the Hamilton cycle. Such a critical pair corresponds to a colored angle at $v$. Since the 
order of each color reverses all critical pairs corresponding to this color each critical pair of this class is reversed.

It remains to prove that all vertex-face pairs $(v, F)$ are reversed. Note that $F$ is an interior face. If $v \in V_{l}$ and $F \subset V_{r} \cup\left\{q_{3}\right\}$, then $(v, F)$ is reversed in blue. Similarly, if $v \in V_{r}$ and $F \subset V_{l} \cup\left\{q_{3}\right\}$, then $(v, F)$ is reversed in green. All the vertices that are incomparable to $F_{4}$ are above $F_{4}$ in red, and $q_{3}$ is above all incomparable faces in green or blue. Hence, we only have to show that $(v, F)$ is reversed when $F$ and $v$ are either both left or both right.

Suppose $v \in V_{l}$. The critical pairs $\left(v, F_{0}\right)$ and $v=p_{2}, q_{2}$ are reversed in blue. The pair $\left(p_{0}, F_{1}\right)$ is reversed in green and $\left(p_{2}, F_{1}\right)$ in blue. The two pairs involving $F_{2}$ are reversed in green and all three pairs with $F_{3}$ in red. The cases where $v \in V_{r}$ are symmetric.

Proposition 20. Let $M$ be a maximal path-like map. Then $\operatorname{dim}\left(\mathbf{P}_{M}\right)=3$ if and only if there is a permissible coloring of the chordal edges.

Proof. If $\operatorname{dim}\left(\mathbf{P}_{M}\right)=3$, then there is a permissible coloring of the chordal edges. In view of Lemma 19 we only have to prove that the operations of Lemma 18 do not increase the dimension.

(i): By symmetry we only have to consider the contraction of $\left\{q_{2}, q_{3}\right\}$. The new merged vertex $q_{2,3}$ takes the place of $q_{2}$ in green and blue, and the place of $q_{3}$ in red.

The vertex-edge pairs involving $q_{2,3}$ are reversed in green and red. Now, $q_{2,3}$ is only below the old position of $q_{2}$ in red. But the only vertex-face critical pair with $q_{2}$ that was reversed in red is $\left(q_{2}, F_{4}\right)$, and $q_{2,3} \in F_{4}$, so all critical pairs involving $q_{2,3}$ are reversed.

Now, the position of a face in a partial order can only change if it contains $q_{2}$ or $q_{3}$ as its highest vertex. The new vertex $q_{2,3}$ is as high as $q_{2}$ in green and blue, and as high as $q_{3}$ in red, so the only affected face is $F_{5}$ in blue. In the blue partial order, the critical pairs $\left(v, F_{5}\right), v \in\left\{p_{0}, p_{1}, q_{1}\right\}$, are not reversed anymore. This is taken care of by moving $q_{1}$ (and hence $p_{1}$ and $p_{0}$ ) above $q_{4}$ in red. Since none of $p_{0}, p_{1}$ and $q_{1}$ were comparable to $q_{4}$ in red before, all previously reversed critical pairs are still reversed.

(ii): The partial orders are constructed as before (with possible changes resulting from a $q$-edge contraction). By an argument similar to the proof of Lemma 19, $\operatorname{dim}\left(\mathbf{P}_{M}\right)=3$.

(iii): The only incidence that changes among the remaining elements of $\mathbf{P}_{M}$ is that one chordal edge $e$ now is on the outer face. The edge $e$ is moved below $F_{\infty}$ in its color. The only new critical pair is $(v, e)$, where $v$ is the vertex in the same interior face as $e$ that is not in $e$. But previously, there was a critical pair $(v, F), e \in F$, which was reversed, so $(v, e)$ must be reversed.

We summarize the result in a theorem: 
Theorem 21. Let $M$ be a maximal path-like map. Then the following three conditions are equivalent:

(i) $\operatorname{dim}\left(\mathbf{P}_{M}\right)=3$

(ii) There is a permissible coloring of the chordal edges of $M$.

(ii) $M$ can be obtained from the canonical map via the operations in Lemma 18 .

\subsection{Algorithmic aspects}

Theorem 21 can easily be turned into an algorithm for testing if $\operatorname{dim}\left(\mathbf{P}_{M}\right) \leq$ 3 for a maximal path-like map $M$. Start by fixing one of the two possible orientations and a color some chordal edge. This induces an angle coloring in the adjacent triangles (Lemma 11, Lemma 10). Lemma 12 now gives us the colors of the angles in all the interior triangles in $M$, which in turns induces an oriented coloring of the chordal edges. Hence, given a fixed orientation of one chordal edge, any permissible coloring is unique up to permutations of the colors. To test for $\operatorname{dim}\left(\mathbf{P}_{M}\right) \leq 3$, we check if any vertex has four outgoing edges or if any two bicolored faces have the same colors. This can be done in linear time.

Once we have a permissible coloring of the chordal edges of $M$ a 3-realizer can be generated: Since we now know which vertices are sinks, we know the $p$-edges and $q$-edges of the colored map $M$ and can identify the operations of Lemma 18 (contracting a $q$-edge, subdividing a $q$-edge and removing a part of the map) that have to be applied to get $M$ from the canonical map. The proof of Lemma 19 gives us a 3-realizer of the canonical map, and the proof of Proposition 20 demonstrates how to modify the 3-realizer for each of the operations. This yields an algorithm to produce a 3-realizer of $\mathbf{P}_{M}$ from a permissible coloring of $M$. It is clear from the proofs of Lemma 19 and Proposition 20 that the running time of this algorithm can be bounded by some constant times the number of elements in $\mathbf{P}_{M}$. Since $M$ is a planar map, we have the following theorem.

Theorem 22. There is an algorithm running in time $O(n)$, which takes as input a maximal path-like map $M$ with $n$ vertices and either returns a 3-realizer of $\mathbf{P}_{M}$, or certifies that $\operatorname{dim}\left(\mathbf{P}_{M}\right)=4$.

\section{Vertex-face posets of dimension at most 3}

From Theorem 3 we know that if $\operatorname{dim}\left(\mathbf{Q}_{M}\right) \leq 3$, then $M$ does not contain a subdivision of $K_{4}$. Figure 15 shows an example of a planar map which contains no $K_{4}$-subdivision but still $\operatorname{dim}\left(\mathbf{Q}_{M}\right)=4$. This example from [2], has a dual map which is a $K_{2,3}$, with each edge replaced by a 2 -face. More generally every map $M$ where we can find fences of vertices and faces like in the proof of Theorem 5 must have $\operatorname{dim}\left(\mathbf{Q}_{M}\right)=4$. 


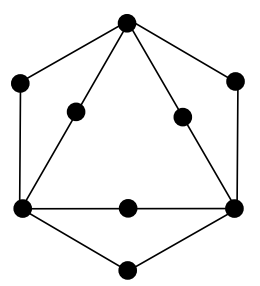

Figure 15: A planar map with vertex-face poset dimension 4.

However, unlike in the vertex-edge-face case, there are no 2-connected maps $M$ of dimension 4 such that both $M$ and $M^{*}$ are weakly outerplanar.

Theorem 23. Let $M$ be a simple 2-connected planar map such both $M$ and its dual $M^{*}$ are weakly outerplanar. Then $\operatorname{dim}\left(\mathbf{Q}_{M}\right) \leq 3$.

Proof. We may assume that no two vertices $v, w$ of degree 2 are neighbors in $M$ : Two such vertices correspond to elements with identical comparabilities in $\mathbf{Q}_{M}$, so called twins. Hence, contracting the edge $v w$ in the graph does not affect $\operatorname{dim}\left(\mathbf{Q}_{M}\right)$.

From Corollary 9, we know that if all chordal edges of $M$ are moved inside the Hamilton cycle, the dual of the resulting map $M^{\prime}$ is $K_{2,3}$-subdivision free. Hence, $M^{\prime}$ is a path-like map. We will inductively construct two linear extensions, $L_{1}$ and $L_{2}$, of $\mathbf{Q}_{M}$, in which all vertex-face pairs are reversed. Similar to the proof of Proposition 7 we start with the path-like map $M_{0}=$ $M^{\prime}$ and then move the required chordal edges outside the Hamilton cycle one by one, creating a series of maps $M_{0}, M_{1}, \ldots, M_{k}=M$.

The case $M_{0}=K_{3}$ is trivial, otherwise, the path-like map $M_{0}$ has exactly two faces that contains only one chordal edge. Each of these two faces contains a vertex of degree 2. Let these two vertices be $\ell$ and $r$.

Given a face $F$ and a vertex $x$, if there is an $x-\ell$ path avoiding $F$, then we say that $x$ is left of $F$. Symmetrically, $x$ is right of $F$ if there is an $x-r$ path avoiding $F$. This definition of left of and right of coincides with the intuition of left and right based on a drawing where the Hamilton cycle is a circle, and $\ell$ and $r$ are its left and right extreme points. Note that $x$ is neither right, nor left of $F$ if and only if $x \in F$. We define a vertex to be to the left (right) of a chordal edge in the same way.

Next, we inductively construct two linear extensions $L_{1}^{i}$ and $L_{2}^{i}$ of the vertex-face poset of $M_{i}$, for $i=0,1, \ldots, k$, such that $L_{1}^{k}=L_{1}$ and $L_{2}^{k}=$ $L_{2}$. Since $M_{0}$ is path-like, the interior dual of $M_{0}$ is a path. In the linear extensions $L_{1}^{0}$ and $L_{2}^{0}$ we order the interior faces by their position in this path, with the face containing $\ell$ highest in $L_{1}^{0}$ and the face containing $r$ highest in $L_{2}^{0}$. The vertices and the outer face are inserted as high as possible.

Let $e$ be the chordal edge that is moved outside the Hamilton cycle when $M_{i}$ is changed to $M_{i+1}$. Before it is moved, it is contained in the two faces $F^{\prime}$ and $F^{\prime \prime}$ that are inside the Hamilton cycle. Let $F^{\prime}$ be the left one, i.e., 
let $F^{\prime}$ contain a vertex $u$ that is left of $e$. When $e$ is moved outside, some face $G$ outside the Hamilton cycle is split into two faces $G^{\prime}$ and $G^{\prime \prime}$. Let $G^{\prime}$ contain $u$. The faces $F^{\prime}$ and $F^{\prime \prime}$ are merged into a new face $F^{+}$(see Figure 16).

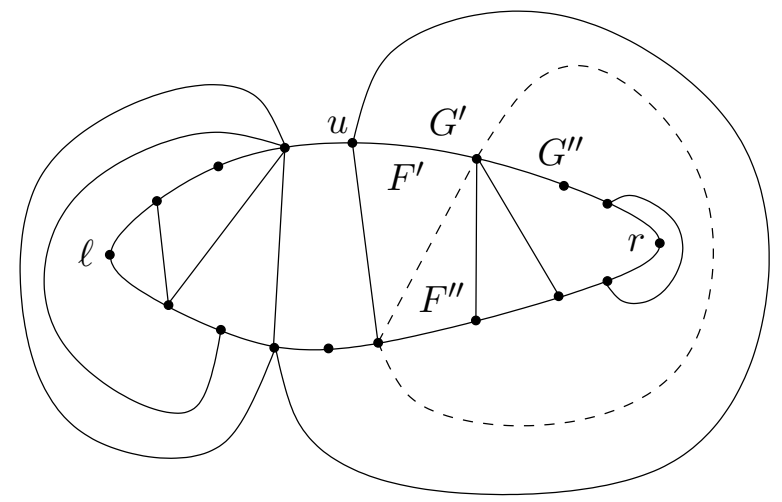

Figure 16: Moving the dashed edge from the inside to the outside.

We can now create $L_{1}^{j+1}$ and $L_{2}^{j+1}$ from $L_{1}^{j}$ and $L_{2}^{j}$. In $L_{1}^{j+1}, F^{+}$is inserted in the position of $F^{\prime}$ in $L_{1}^{j}, G^{\prime}$ is inserted in the position of $G$ and $G^{\prime \prime}$ is inserted in the position of $F^{\prime \prime}$. In $L_{2}^{j+1}, F^{+}$is inserted in the position of $F^{\prime \prime}$ in $L_{2}^{j}$, and $G^{\prime \prime}$ and $G^{\prime}$ are inserted in the positions of $G$ and $F^{\prime}$, respectively.

Claim A. For $i=0,1, \ldots, k, L_{1}^{i}$ and $L_{2}^{i}$ are linear extensions of $\mathbf{Q}_{M_{i}}$. If a vertex $v$ is to the left of a face $F$ in the map $M_{i}$, then $v>F$ in $L_{1}^{i}$, and if $v$ is to the right of $F, v>F$ in $L_{2}^{i}$.

The claim can be verified by induction on $i$. From the construction of $L_{1}^{0}$ and $L_{2}^{0}$, it is clear that the claim is true for $i=0$.

Suppose the claim is true for $i$. The map $M^{i+1}$ is constructed by moving the edge $e$ in $M_{i}$ outside the Hamilton cycle. To verify that $L_{1}^{i+1}$ and $L_{2}^{i+1}$ are linear extensions of $\mathbf{Q}_{M_{i+1}}$ it is enough to check that $F, G^{\prime}$ and $G^{\prime \prime}$ are above all the vertices contained in them. This is immediate from the construction.

It remains to prove the second part of the claim. By induction and symmetry it is enough to consider the case where $v$ is to the left of $e$ and $F$ is one of $F^{+}, G^{\prime}$ and $G^{\prime \prime}$. If $v$ is to the left of $e$, either $v \in F^{\prime}$ or $v$ is to the left of $F^{\prime}$. In the latter case $v$ is above $F^{\prime}$ in $L_{1}^{i}$, so $v>F^{+}$in $L_{1}^{i+1}$. If $v$ is to the left of $G$, then $v$ is also to the left of $G^{\prime}$ and $G^{\prime \prime}$. Since $v>G$ in $L_{1}^{i}$, $v>G^{\prime}>G^{\prime \prime}$ in $L_{1}^{i+1}$. On the other hand, if $v \in G, v$ must also be in $G^{\prime}$ and to the left of $G^{\prime \prime}$. But $v$ is to the left of $F^{\prime \prime}$, so $v>F^{\prime \prime}$ in $L_{1}^{i}$ by construction, and hence $v>G^{\prime \prime}$ in $L_{1}^{i+1}$. Hence, the claim is true for $i+1$.

Claim A implies that all vertex-face critical pairs of $\mathbf{Q}_{M}$ are reversed in $L_{1}$ and $L_{2}$. It remains is to find a linear extension $L_{3}$ of $\mathbf{Q}_{M}$ which reverses 
all vertex-vertex and face-face critical pairs. Such a linear extension can be obtained by taking all faces above all vertices. The order of the faces in $L_{3}$ is choosen as the reverse of the order of faces in $L_{1}$ and alike the order of vertices is reversed between $L_{3}$ and $L_{1}$. Together $L_{1}, L_{2}, L_{3}$ reverse all critical pairs. Hence, they form a realizer and $\operatorname{dim}\left(\mathbf{Q}_{M}\right) \leq 3$.

In a strongly outerplanar map $M$ it is never the case that we can find fences like in the proof of Theorem 5. The interior dual is a tree, and $F_{\infty}$ contains all the vertices. Hence, $F_{\infty}^{*}$ has to be one of the degree 3 -vertices of any $K_{2,3}$-subdivision in $M^{*}$ ( $M$ contains no $K_{2,3}$-subdivision since it is outerplanar). Therefore, the existence of a strongly outerplanar map of vertex-face dimension 4 is not obvious.

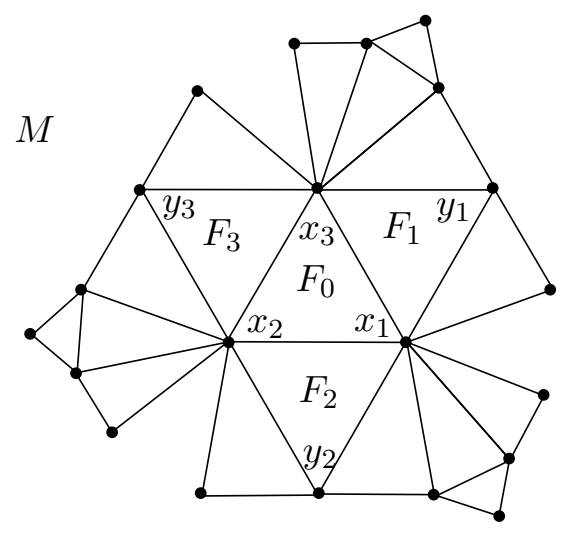

Figure 17: An outerplanar map with $\operatorname{dim}\left(\mathbf{Q}_{M}\right)=4$.

Theorem 24. The strongly outerplanar map $M$ shown in Figure 17 has a vertex-face poset of dimension four.

Proof. Suppose $\operatorname{dim}\left(\mathbf{Q}_{M}\right) \leq 3$. Again we identify the three linear extensions of a realizer with three colors and use these to color critical pairs. Our main focus will be on the coloring of critical pairs involving a vertex and an interior face.

Fact A. For an interior face $F$ only two colors appear at critical pairs $(v, F)$. Suppose that the critical pair $\left(F, F_{\infty}\right)$ is reversed in color $i$. This forces all vertices below $F$ in color $i$, and hence all critical pairs $(v, F)$ are reversed in the other two colors.

Fact B. If a triangular face $\Delta=\left\{v_{1}, v_{2}, v_{3}\right\}$ is surrounded by interior faces $F_{1}, F_{2}, F_{3}$ such that $v_{i} \notin F_{i}$, then the three critical pairs $\left(v_{i}, F_{i}\right)$ use all three colors. 
Any two of the three critical pairs form an alternating cycle and, hence, require different colors. Equivalently, the order induced by $v_{1}, v_{2}, v_{3}, F_{1}, F_{2}, F_{3}$ is a 3 -crown.

These two facts are applied to the central face $F_{0}$ of the map $M$ : Fact B implies that the three critical pairs $\left(x_{i}, F_{i-1}\right)$ use all three colors. Symmetry among the colors allows us to assume that $\left(x_{2}, F_{1}\right)$ is red, $\left(x_{3}, F_{2}\right)$ green and $\left(x_{1}, F_{3}\right)$ blue. Fact $\mathrm{A}$ implies that two of the three critical pairs $\left(y_{i}, F_{0}\right)$ have the same color. The symmetry of the graph allows to assume that this duplicated color is blue. It is infeasible to have $\left(y_{3}, F_{0}\right)$ in blue, because it forms an alternating cycle with the blue pair $\left(x_{1}, F_{3}\right)$. Hence, $\left(y_{1}, F_{0}\right)$ and $\left(y_{2}, F_{0}\right)$ are both blue.

To reach a contradiction we can from now on concentrate on the submap of $M$ shown in Figure 18. The colors of critical pairs which have already been fixed are indicated by the colored arrows in the figure.

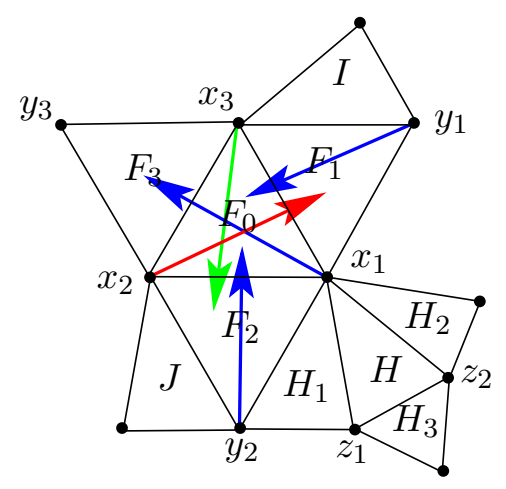

Figure 18: Having fixed the colors of some critical pairs we concentrate on a submap $M^{\prime}$ of $M$.

To avoid a monochromatic alternating cycle with the blue pair $\left(y_{1}, F_{0}\right)$ and with the green pair $\left(x_{3}, F_{2}\right)$ the color of $\left(x_{1}, I\right)$ has to be red. Similarly, the colors of $\left(y_{2}, F_{0}\right)$ and $\left(x_{2}, F_{1}\right)$ imply that $\left(x_{1}, J\right)$ is green.

From the critical pairs $\left(x_{1}, J\right)$ and $\left(x_{1}, F_{3}\right)$ we know that $x_{1}>x_{2}$ in green and blue, so all critical pairs $\left(x_{2}, F\right)$, where $x_{1} \in F$, must be red. Similarly, all critical pairs $\left(x_{3}, F\right)$, where $x_{1} \in F$, must be green. In particular we have $\left(x_{2}, H_{i}\right)$ red and $\left(x_{3}, H_{i}\right)$ green for $i=1,2$.

From Fact A applied to $H_{1}$ and $H_{2}$ we can conclude that neither $\left(z_{1}, H_{2}\right)$ nor $\left(z_{2}, H_{1}\right)$ can be blue. Applying Fact $\mathrm{B}$ to face $H$ we can conclude that $\left(x_{1}, H_{3}\right)$ is blue. See Figure 19.

Consider the critical pair $\left(z_{1}, F_{0}\right)$. It forms an alternating cycle with $\left(x_{1}, H_{3}\right)$, hence it can't be blue. It forms an alternating cycle with $\left(x_{2}, H_{1}\right)$, hence it can't be red. It forms an alternating cycle with $\left(x_{3}, H_{1}\right)$, hence it can't be green. Consequently there is no legal 3-coloring of the hypergraph of critical pairs of $\mathbf{Q}_{M}$. 


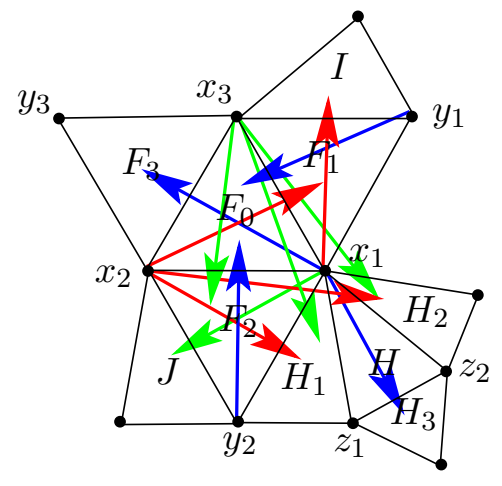

Figure 19: Colored critical pairs on $M^{\prime}$.

The maximal outerplanar map $T_{4}$ shown in Figure 20 has a vertex-face poset of dimension 3 (a 3-realizer is listed in Table 4). This suggests that the example of a strongly outerplanar map $M$ with $\operatorname{dim}\left(\mathbf{Q}_{M}\right)=4$ given in Theorem 24 is close to a minimal example. Figure 21 shows a map where all 2-connected components are submaps of $T_{4}$ and hence have vertex-face poset dimension 3. Still an argument as in Theorem 24] shows that the map in Figure 21 has a 4-dimensional vertex-face poset.

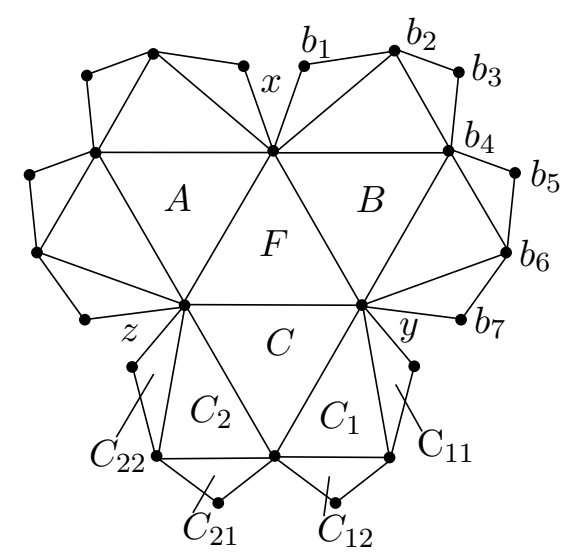

Figure 20: A 2-connected outerplanar map with vertex-face dimension 3. The naming scheme of the faces and vertices of the map is indicated in the figure.

\section{Concluding remarks}

When we started our investigations we set out to characterize the planar maps with vertex-edge-face posets of dimension at most 3 . We proved that for all such maps $M$, both $M$ and its dual $M^{*}$ must be weakly outerplanar. In the case of maximal path-like maps, we found necessary and sufficient 


\begin{tabular}{ccccccccc}
$L_{1}^{1}$ & $L_{2}^{1}$ & $L_{3}^{1}$ & $L_{1}^{2}$ & $L_{2}^{2}$ & $L_{3}^{2}$ & $L_{1}^{3}$ & $L_{2}^{3}$ & $L_{3}^{3}$ \\
\hline$A_{22}$ & $C$ & $C_{11}$ & $B_{2}$ & $F$ & $c_{7}$ & $c_{7}$ & $a_{1}$ & $b_{7}$ \\
$A_{2}$ & $B_{12}$ & $C_{1}$ & $b_{4}$ & $C_{11}$ & $a_{2}$ & $C_{2}$ & $A_{1}$ & $B_{2}$ \\
$A$ & $B_{21}$ & $C_{12}$ & $B_{22}$ & $c_{1}$ & $a_{1}$ & $C$ & $A$ & $B$ \\
$F$ & $B_{2}$ & $C_{21}$ & $b_{6}$ & $C_{1}$ & $a_{3}$ & $z$ & $z$ & $y$ \\
$B$ & $B_{22}$ & $C_{2}$ & $b_{7}$ & $y$ & $a_{5}$ & $C_{21}$ & $A_{12}$ & $B_{21}$ \\
$B_{1}$ & $F_{\infty}$ & $C_{22}$ & $A_{21}$ & $C_{12}$ & $C$ & $c_{6}$ & $a_{2}$ & $b_{6}$ \\
$B_{11}$ & $b_{6}$ & $A_{11}$ & $a_{6}$ & $c_{2}$ & $c_{4}$ & $c_{5}$ & $a_{3}$ & $b_{5}$ \\
$F_{\infty}$ & $b_{3}$ & $A_{1}$ & $a_{5}$ & $c_{3}$ & $A_{22}$ & $C_{12}$ & $A_{21}$ & $B_{12}$ \\
$x$ & $b_{5}$ & $A_{12}$ & $A_{12}$ & $C_{21}$ & $a_{7}$ & $c_{3}$ & $a_{5}$ & $b_{3}$ \\
$a_{7}$ & $b_{7}$ & $A_{21}$ & $a_{3}$ & $c_{5}$ & $A_{2}$ & $C_{1}$ & $A_{2}$ & $B_{1}$ \\
$b_{1}$ & $B_{11}$ & $F_{\infty}$ & $A_{1}$ & $C_{2}$ & $a_{6}$ & $c_{4}$ & $a_{4}$ & $b_{4}$ \\
$B_{12}$ & $b_{1}$ & $c_{2}$ & $a_{4}$ & $c_{4}$ & $A$ & $C_{11}$ & $A_{22}$ & $B_{11}$ \\
$b_{2}$ & $B_{1}$ & $c_{6}$ & $A_{11}$ & $C_{22}$ & $a_{4}$ & $c_{1}$ & $x$ & $x$ \\
$b_{3}$ & $b_{2}$ & $c_{1}$ & $a_{2}$ & $c_{6}$ & $F$ & $c_{2}$ & $a_{6}$ & $b_{2}$ \\
$B_{21}$ & $B$ & $c_{3}$ & $a_{1}$ & $c_{7}$ & $z$ & $c_{1}$ & $a_{7}$ & $b_{1}$ \\
$b_{5}$ & $b_{4}$ & $c_{5}$ & $C_{22}$ & $A_{11}$ & $B_{22}$ & & &
\end{tabular}

Table 1: A 3-realizer of the map in Figure 20. The order $L_{i}$ is obtained from the concatenation $L_{i}=L_{i}^{1} \oplus L_{i}^{2} \oplus L_{i}^{3}$.

conditions for dimension at most 3 using an oriented coloring of the chordal edges. What remains open are four cases:

- $M$ is path-like but not maximal, i.e., has non-triangular faces.

If $M$ can be obtained from a maximal path-like map $M_{0}$ by subdividing some edges of the Hamilton cycle, then $\operatorname{dim}\left(\mathbf{P}_{M}\right)=\operatorname{dim}\left(\mathbf{P}_{M_{0}}\right)$. To see this, consider a 2-connected map $M$ with $\operatorname{dim}\left(\mathbf{P}_{M}\right) \leq 3$ and an edge $e=\{u, v\}$ of the Hamilton cycle. Let edge $e$ be subdivided in the new map $M^{\prime}$ by a vertex $w$ incident to the new cycle edges $e_{1}=\{u, w\}$ and $e_{2}=\{w, v\}$. We can modify a 3-realizer of $\mathbf{P}_{M}$ to obtain realizer of $\mathbf{P}_{M^{\prime}}$ in the following way.

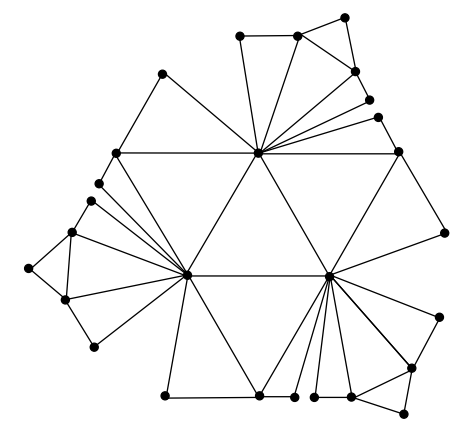

Figure 21: A map $M$ with $\operatorname{dim}\left(\mathbf{Q}_{M}\right)=4$, where each 2-connected component $C$ has $\operatorname{dim}\left(\mathbf{Q}_{C}\right)=3$ 
In each linear extension, insert $e_{1}$ in the old position of $e$ if $v<u$, and right below $v$ if $v>u$. Symmetrically, $e_{2}$ is inserted into the position of $e$ if $u<v$, and right below $u$ if $u>v$. The vertex $w$ is inserted right below $\min \left\{e_{1}, e_{2}\right\}$. This produces a 3 -realizer of $\mathbf{P}_{M^{\prime}}$. The general case remains a challenge.

- $M$ is simple, and both $M$ and $M^{*}$ are weakly outerplanar, but neither of them is strongly outerplanar.

We can extend the coloring approach from Section 3 to get a set of necessary conditions for $\operatorname{dim}\left(\mathbf{P}_{M}\right) \leq 3$ in this case. There is a natural way to get an oriented coloring as in the case of path-like maps, if $M$ is a different drawing of the graph of some maximal path-like map. Instead of coloring the angles of triangular faces, we color the angles of triangles in the strongly outerplanar drawing of the graph of $M$. Again, this angle coloring can be encoded as an oriented coloring of the chordal edges. Instead of each vertex being a sink or a source, each vertex will now be a sink on one side of the Hamilton cycle and a source on the other side. The proof of this is similar to the proof of Lemma 14.

If $F$ is a face in the 2-connected map $M$ with $\operatorname{dim}\left(\mathbf{P}_{M}\right) \leq 3$, the submap $M_{F}$ induced by the vertices in $F$ must be path-like by Theorem 5 , In the same way, the submap $M_{v^{*}}^{*}$ of the dual map $M^{*}$ induced by the dual vertices in the dual face $v^{*}$ is also path-like. Since $(x, y)$ is a critical pair in $\mathbf{P}_{M}$ if and only if $\left(y^{*}, x^{*}\right)$ is a critical pair in $\mathbf{P}_{M^{*}}$, the primal oriented coloring induces an oriented coloring in the dual map. Hence, the oriented coloring of a map $M$ with $\operatorname{dim}\left(\mathbf{P}_{M}\right) \leq 3$ must be permissible "locally" around each vertex and face. The question remains whether the existence of such a locally permissible coloring is also a sufficient condition for $\operatorname{dim}\left(\mathbf{P}_{M}\right) \leq 3$, or if there are some non-local effects that force $\operatorname{dim}\left(\mathbf{P}_{M}\right)=4$.

- $M$ is not simple.

- $M$ is not 2-connected.

Suppose $M$ is not 2-connected. From $\operatorname{dim}\left(\mathbf{P}_{C}\right) \leq 3$ for each 2-connected component $C$ it can not be concluded that $\operatorname{dim}\left(\mathbf{P}_{M}\right) \leq 3$. The conclusion is not even possible if all components are maximal path-like maps and have a common outer face. Consider the map $M$ constructed by taking two maximal path-like maps $C_{1}$ and $C_{2}$ and identifying two vertices $v_{1} \in C_{1}$ and $v_{2} \in C_{2}$ and the outer faces of each map. We choose $C_{1}$ and $C_{2}$ such that $\operatorname{dim}\left(\mathbf{P}_{C_{1}}\right)=\operatorname{dim}\left(\mathbf{P}_{C_{2}}\right)=3$ and that in any permissible coloring of the chordal edges in each map $C_{i}$ there will be two outgoing edges from $v_{i}$. Such maps clearly exist. A straightforward modification of Proposition [15] will now show that $\operatorname{dim}\left(\mathbf{P}_{M}\right)=4$.

\section{Vertex-face posets and posets of height 2}

For vertex-face posets, we saw that it seems hard to characterize even the strongly outerplanar 2-connected maps with dimension at most 3 . This 
relates to the long-standing open question if it is NP-hard to determine if the dimension of a height 2 poset is at most 3. Yannakakis [1] proved in 1982 that it is NP-hard to determine if $\operatorname{dim}(\mathbf{P}) \leq 3$ for posets $\mathbf{P}$ of height at least 3. Brightwell and Trotter [2] refined the question and asked if it is NP-hard to recognize planar maps with $\operatorname{dim}\left(\mathbf{Q}_{M}\right) \leq 3$. Given our results, it makes sense to ask this question even for 2-connected strongly outerplanar maps.

\section{Acknowledgment}

We thank Graham Brightwell for stimulating discussions and a referee for comments that helped improve the paper.

\section{References}

[1] G. Brightwell and W. Trotter, The order dimension of convex polytopes, SIAM J. Discret. Math., 6 (1993), pp. 230-245.

[2] G. R. Brightwell and W. T. Trotter, The order dimension of planar maps, SIAM J. Discret. Math., 10 (1997), pp. 515-528.

[3] B. Dushnik And E. Miller, Partially ordered sets, Am. J. Math., 63 (1941), pp. $600-610$.

[4] S. Felsner And W. Trotter, Posets and planar graphs, J. Graph Th., 49 (2005), pp. 262-272.

[5] S. Felsner And F. Zickfeld, On the number of planar orientations with prescribed degrees, Electron. J. Comb, 15, No. 1, R77 (2008). 41 pages.

[6] S. MitchelL, Linear algorithms to recognize outerplanar and maximal outerplanar graphs, Inform. Process. Lett., 9 (1979), pp. 229-232.

[7] R. MöHring, Computationally tractable classes of ordered sets, in Algorithms and Orders, I. Rival, ed., Reidel Dordrecht, 1989, pp. 105-194.

[8] C. M. L. (SARAh), 2008. personal communication.

[9] W. SChNyder, Planar graphs and poset dimension, Order, 5 (1989), pp. 323343.

[10] W. T. Trotter, Combinatorics and partially ordered sets, Johns Hopkins Univ. Press, 1992.

[11] M. YANNAKAKIS, The complexity of the partial order dimension problem, SIAM J. Algebraic Discrete Methods, 3 (1982), pp. 351-358. 